ISSN electrónico: 2445-1355

DOI: https://doi.org/10.14201/fj2021626373

\title{
TERAPIA ANTIPLAQUETARIA EN CÁNCER
}

\section{Antiplatelet therapy in Cancer}

\author{
Alfonso TORICES*; Carmen GUERRERO** \\ *Hospital CUF Descobertas, 1998-018, Lisboa, Portugal \\ *Centro de Investigación del Cáncer, IBMCC, CSIC-USAL. Departamento de \\ Medicina. Universidad de Salamanca, Salamanca, España. \\ Autor de correspondencia: Carmen Guerrero. Centro de Investigación del Cáncer. \\ Campus Unamuno, s/n. \\ 37007 Salamanca. España.
}

Correo-e: cguerrero@usal.es**

RESUMEN: Las plaquetas contienen en su interior gránulos con sustancias proangiogénicas y antiangiogénicas. En el microambiente tumoral las plaquetas interaccionan con las células neoplásicas, las cuales liberan agonistas que inducen la activación y la agregación plaquetaria, así como la secreción de factores proangiogénicos que favorecen la vascularización y el crecimiento tumoral. Además, las plaquetas protegen a las células tumorales del sistema inmune y promueven la transición epitelio-mesenquimal, aumentando su capacidad invasiva y favoreciendo la aparición de metástasis. Existen numerosos estudios que muestran el beneficio de la terapia antiplaquetaria en la reducción de metástasis. De hecho, la aparición de tromboembolismos durante el desarrollo neoplásico es un factor de mal pronóstico. En este trabajo, hemos comparado la progresión tumoral de pacientes con cáncer colorrectal (CCR) tratados con terapia antiplaquetaria con pacientes con la misma neoplasia pero sin tratamiento antiplaquetario. Nuestros resultados mostraron que el $90 \%$ de los pacientes de CCR tratados con antiplaquetarios no desarrollaron metástasis, frente al 59,8\% de los pacientes sin dicha terapia. El análisis estadístico de los datos indicó una correlación significativa entre un alto recuento plaquetario y el desarrollo de metástasis. Por tanto,

Ediciones Universidad de Salamanca / @@ఠ $\quad$ FarmaJournal, vol. 6, núm. 2 (2021), pp. 63-73 
la terapia antiplaquetaria en cáncer podría ser beneficiosa y complementar la terapia antineoplásica convencional.

Palabras clave: Plaquetas; cáncer; terapia antiplaquetaria; metástasis; angiogénesis.

ABSTRACT: Platelets contain granules with proangiogenic and antiangiogenic substances. In the tumor microenvironment, platelets interact with neoplastic cells, which release agonists that induce platelet activation and aggregation, as well as the secretion of proangiogenic factors that favor vascularization and tumor growth. In addition, platelets protect tumor cells from the immune system and promote the epithelial-mesenchymal transition, increasing their invasive capacity and favoring the appearance of metastasis. There are numerous studies showing the benefit of antiplatelet therapy in reducing metastases. In fact, the appearance of thromboembolisms during neoplastic development is a poor prognostic factor. In this work, we have compared the tumor progression of patients with colorectal cancer (CRC) treated with antiplatelet therapy with that of patients with the same neoplasm but without antiplatelet treatment. Our results showed that $90 \%$ of the CRC patients treated with antiplatelet therapy did not develop metastases, compared to $59.8 \%$ of the patients without such therapy. Statistical analysis of the data indicated a significant correlation between a high platelet count and the development of metastases. Therefore, antiplatelet therapy in cancer could be beneficial and complement conventional antineoplastic therapy.

Keywords: Platelets; Cancer; Antiplatelet therapy; Metastasis; angiogenesis.

\section{INTRODUCCIÓN}

\subsection{Generalidades}

La relación entre las plaquetas y el cáncer está bien establecida, ya que aproximadamente el $20 \%$ de los pacientes oncológicos desarrollan un episodio tromboembólico a lo largo de la historia natural de la neoplasia, siendo la trombosis la segunda causa de muerte en estos pacientes. Por esta razón es importante entender el papel de las plaquetas en el cáncer.

Las plaquetas son pequeñas células anucleadas generadas a partir de megacariocitos en la médula ósea. Ejercen una función hemostática en el organismo evitando la aparición de hemorragias tras la ruptura de un vaso sanguíneo. En su interior contienen gránulos- $\alpha$ con diversos factores proangiogénicos como factor 
de crecimiento endotelial vascular (VEGF), factor de crecimiento epidérmico (EGF), factor de crecimiento derivado de plaquetas (PDGF), factor de crecimiento transformante $\beta$ (TGF $\beta$ ), angiogenina y metaloproteinasas de matriz (MMPs). Pero también contienen gránulos con factores antiangiogénicos como endostatina, factor plaquetario 4 (PF4), trombospondina-1, angiostatina, inhibidor del activador de plasminógeno 1 y $\alpha$-macroglobulina (Italiano et al., 2008). El equilibrio entre el contenido de los dos tipos de gránulos- $\alpha$ determina la función que ejercerán las plaquetas, actuando como anti- o proangiogénicas.

\subsection{Activación e interacción plaqueta-célula tumoral}

El papel de las plaquetas en el desarrollo de procesos tumorales se comenzó a documentar hace casi 60 años con estudios que relacionaban trombocitosis con aparición de procesos neoplásicos (Levin y Conley, 1964).

Cuando las plaquetas se encuentran en el microambiente tumoral y entran en contacto con las células cancerígenas, estas son capaces de activar a las plaquetas, lo que va a favorecer la proliferación de clones tumorales, obteniendo resistencia a apoptosis e induciendo angiogénesis (Xu et al., 2018). Las células cancerígenas son capaces de liberar agonistas plaquetarios como ADP o trombina, los cuales producen estimulación y activación de las plaquetas. Tras dicha activación las plaquetas modulan su perfil de ARN de forma que sintetizan sustancias procoagulantes y proangiogénicas, que favorecen el crecimiento y la progresión tumoral. Estas plaquetas reciben el nombre de "plaquetas educadas por el tumor" (tumor educated platelets, TEP) (Xu et al., 2018).

Posteriormente las plaquetas interaccionan con las células neoplásicas a través de moléculas como la caderina-6, induciendo la formación de micropartículas derivadas de las plaquetas (PMP). Las PMP pueden contener diversas sustancias como micro-ARN, INFy, IL-4, CCL2, CXCL12, receptores plaquetarios o incluso lípidos como ácido araquidónico, produciendo disminución o aumento del crecimiento tumoral en función del tipo de sustancia (Contursi et al., 2018).

Tras la interacción plaqueta-célula tumoral, las plaquetas pueden captar exosomas que contienen ARN tumoral, los cuales se funden con la membrana plaquetaria, modulando el contenido de ARN de las plaquetas. Dicho ARN plaquetario puede ser analizado sirviendo como biomarcador de distintos tipos de cánceres en estadios tempranos (Menter et al., 2014).

Las células tumorales inducen además un aumento de la producción de trombopoyetina a nivel hepático a través de la secreción de IL-6 y pueden activar directamente la cascada de coagulación. La expresión de factor tisular (TF) en células cancerígenas produce activación plaquetaria (Cedervall et al., 2018) y, a su vez, las plaquetas activadas producen una mayor cantidad de pre-ARNm de 
TF. Este aumento de la concentración de TF favorece el crecimiento y la estabilización de trombos, dando lugar a la aparición de tromboembolismos venosos y contribuyendo a un mal pronóstico (Suzuki-Inoue, 2019).

\subsection{Plaquetas, crecimiento tumoral y metástasis}

Tras un daño tisular, la activación plaquetaria es el primer evento que tiene lugar para garantizar la reparación del tejido dañado. Sin embargo, si esa respuesta no tiene lugar de forma controlada, puede contribuir a la aparición de una respuesta inflamatoria crónica a través de la liberación de mediadores celulares y factores de crecimiento. La expresión de P-selectina en la superficie de las plaquetas tras su activación promueve extravasación de células tumorales ya que favorece su adhesión a las células del endotelio (Ivanov et al., 2019).

Además, las plaquetas activadas producen mediadores lipídicos (tromboxano, prostaglandinas), ADP, factores de crecimiento y microvesículas (conteniendo ARN y micro-ARN) que inducen cambios tanto en las células tumorales como en células del estroma y del sistema inmune. Las células del estroma secretan factores de crecimiento y mediadores inflamatorios que favorecen el desarrollo tumoral. La interacción de las plaquetas con las células del sistema inmune compromete su supervivencia y, por tanto, su respuesta a las células cancerosas (Contursi et al., 2018).

Asimismo, las plaquetas contribuyen a la diseminación de las células cancerígenas a través de la liberación de factores de crecimiento, como VEGF o TGFß. La activación sinérgica de la señalización celular dependiente de TGFß/Smad y $\mathrm{NF}-\kappa \mathrm{B}$ inducida por contacto directo de células tumorales con plaquetas promueve la transición epitelial-mesenquimal (EMT), aumentando la capacidad invasiva de las células tumorales y su proliferación, siendo esta transición un prerrequisito para la aparición de metástasis (Labelle et al., 2011; Contursi et al., 2018).

Las TEP también son capaces de formar agregados plaquetarios que rodean a las células tumorales protegiéndolas del sistema inmune. Además, la interacción plaqueta-leucocito modula el reconocimiento de las células tumorales por parte del sistema inmune (Stoiber y Assinger, 2020).

Por otro lado, estudios recientes han demostrado que las plaquetas pueden transferir sus moléculas de complejo mayor de histocompatibilidad de clase I a las células cancerígenas, promoviendo su capacidad de escapar al sistema inmune (Placke et al., 2012).

Para facilitar su diseminación, las células cancerígenas son también capaces de mimetizar a las plaquetas expresando productos de genes específicos de megacariocitos como GPIIb-IIIa, receptores de proteasa activada (PARs) y moléculas plaquetarias de adhesión a células endoteliales como P-selectinas. 


\subsection{Terapia antiplaquetaria}

El beneficio de la inhibición plaquetaria en el tratamiento antitumoral fue demostrado inicialmente por Gasic et al., en cuyo estudio demuestran la reducción de la formación de metástasis en ratones a través de la inducción de trombocitopenia con neuroaminidasa (Gasic et al., 1968). Diferentes fármacos antiplaquetarios pueden tener posibles acciones terapéuticas en cáncer, tal como se muestra en la Tabla 1.

TABLA 1. Tabla terapia antiplaquetaria con posible acción terapéutica en cáncer.

Adaptado de (Xu et al., 2018)

\begin{tabular}{|c|c|c|}
\hline Fármaco & Mecanismo de acción & Posible aplicación terapéutica \\
\hline $\begin{array}{l}\text { Ácido } \\
\text { acetilsalicílico } \\
\text { (AAS), } \\
\text { Triflusal }\end{array}$ & $\begin{array}{l}\text { Inhibición de las enzimas COX- } \\
1 \text { y COX-2 (Disminución de } \\
\text { tromboxano A2 y prostaglandina } \\
\mathrm{E}_{2} \text { ) } \\
\text { Inhibición de la cascada de } \\
\text { señalización celular NF- } \mathrm{B} \text { y } \\
\text { reducción de la expresión del } \\
\text { receptor TIGIT }\end{array}$ & $\begin{array}{l}\text { Profilaxia de aparición de cáncer } \\
\text { y prevención de desarrollo de } \\
\text { metástasis. }\end{array}$ \\
\hline $\begin{array}{l}\text { Clopidogrel } \\
\text { Prasugrel } \\
\text { Ticagrelor } \\
\text { Ticlopidina } \\
\text { Cangrelor }\end{array}$ & $\begin{array}{l}\text { Inhibición del receptor plaquetario } \\
\text { P2Y12 }\end{array}$ & $\begin{array}{l}\text { Coadministración de Clopidogrel } \\
\text { y AAS mejora la eficacia de la } \\
\text { inmunoterapia celular adoptiva. } \\
\text { Posible uso en la inhibición del } \\
\text { desarrollo tumoral. }\end{array}$ \\
\hline $\begin{array}{l}\text { Abciximab } \\
\text { Tirofibán } \\
\text { Eptifibátida }\end{array}$ & $\begin{array}{l}\text { Inhibición del receptor plaquetario } \\
\text { GP IIb/IIIa }\left(\alpha_{\mathrm{IIb}} \beta 3\right)\end{array}$ & $\begin{array}{l}\text { Inhibición de la metástasis ósea en } \\
\text { el melanoma, control de metástasis } \\
\text { en cáncer de mama e inhibición del } \\
\text { desarrollo tumoral en varios tipos } \\
\text { de neoplasia. }\end{array}$ \\
\hline Anfibatide & $\begin{array}{l}\text { Inhibición del receptor plaquetario } \\
\text { GPIba }\end{array}$ & $\begin{array}{l}\text { Inhibición en la generación de } \\
\text { trombopoyetina y por tanto en la } \\
\text { trombocitosis inducida por tumor. }\end{array}$ \\
\hline $\begin{array}{l}\text { Vorapaxar } \\
\text { Atopaxar }\end{array}$ & $\begin{array}{l}\text { Inhibición del receptor plaquetario } \\
\text { PAR1 }\end{array}$ & $\begin{array}{l}\text { Posible uso en la prevención de } \\
\text { metástasis aumentada por el efecto } \\
\text { de la trombina. }\end{array}$ \\
\hline $\begin{array}{l}\text { Revacept } \\
\text { Losartan }\end{array}$ & $\begin{array}{l}\text { Inhibición del receptor plaquetario } \\
\text { GPVI }\end{array}$ & $\begin{array}{l}\text { Reducción en el desarrollo de } \\
\text { metástasis. }\end{array}$ \\
\hline
\end{tabular}




\section{Materiales y métodos}

\subsection{Descripción del estudio}

Se realizó un estudio observacional de tipo retrospectivo de una muestra de 112 pacientes oncológicos con neoplasia colorrectal. La razón de esta selección es que el CCR es uno de los cánceres más extendidos, con una tasa de incidencia y mortalidad muy elevada. Para ello se accedió a una lista de pacientes con CCR desde el año 2018 hasta el 2020 de la cadena de hospitales CUF (Portugal). Posteriormente, se analizó la historia clínica de cada paciente a través del programa informático GLINTT para realizar una valoración individual sobre la progresión/regresión del estadio tumoral desde que se diagnosticó la neoplasia hasta noviembre de 2020. Para seleccionar y clasificar los pacientes de nuestro estudio se tuvieron en cuenta los siguientes parámetros: evolución plaquetaria, estadio tumoral, función hepática, valores de proteína reactiva C (PCR), leucocitosis, pruebas diagnósticas, farmacología antiplaquetaria, historia clínica.

Una muestra de los pacientes con CCR realizaban tratamiento antiplaquetario con ácido acetisalicílico (AAS), Clopidogrel o Triflusal, desde hace más de 5 años, debido a patologías cardiovasculares concomitantes. En cuanto a la posología en el caso del AAS era de 100-150 mg/día en una única toma, Clopidogrel 75 mg/día en una única toma y Triflusal $600 \mathrm{mg} /$ día en toma única también. El fármaco más usado por los pacientes fue el AAS, seguido por su asociación con Clopidogrel. 


\section{Resultados}

TABla 2. Resultados del contraste de variables aparición de metástasis, recuento de plaquetas por microlitro $(\mu \mathrm{l})$ y tratamiento antiplaquetario

\begin{tabular}{|c|c|c|c|c|c|c|}
\hline \multirow{2}{*}{\multicolumn{4}{|c|}{ Tratamiento antiplaquetario }} & \multicolumn{2}{|c|}{$\begin{array}{l}\text { Recuento de plaquetas } \\
\text { (por microlitro) }\end{array}$} & \multirow{3}{*}{$\begin{array}{l}\text { Total } \\
49 \\
\end{array}$} \\
\hline & & & & \multirow{2}{*}{$\begin{array}{r}<450.000 \\
35\end{array}$} & \multirow{2}{*}{$\begin{array}{r}\geq 450.000 \\
14\end{array}$} & \\
\hline \multirow[t]{9}{*}{ NO } & \multirow{6}{*}{$\begin{array}{l}\text { Aparición } \\
\text { de } \\
\text { metástasis }\end{array}$} & \multirow[t]{3}{*}{$\mathrm{NO}$} & Número de pacientes & & & \\
\hline & & & $\begin{array}{l}\text { \% de pacientes dentro de los } \\
\text { pacientes sin metástasis }\end{array}$ & $71,4 \%$ & $28,6 \%$ & $100,0 \%$ \\
\hline & & & $\begin{array}{l}\% \text { de pacientes en base al } \\
\text { recuento de plaquetas }\end{array}$ & $94,6 \%$ & $31,1 \%$ & \\
\hline & & \multirow[t]{3}{*}{ SÍ } & Número de pacientes & 2 & 31 & 33 \\
\hline & & & $\begin{array}{l}\text { \% de pacientes dentro de los } \\
\text { pacientes con metástasis }\end{array}$ & $6,1 \%$ & $93,9 \%$ & $100,0 \%$ \\
\hline & & & $\begin{array}{l}\% \text { pacientes en base al } \\
\text { recuento de plaquetas }\end{array}$ & $5,4 \%$ & $68,9 \%$ & \\
\hline & \multirow{3}{*}{\multicolumn{2}{|c|}{ Total }} & Número de pacientes & 37 & 45 & 82 \\
\hline & & & $\begin{array}{l}\% \text { de pacientes en base al } \\
\text { número de pacientes totales }\end{array}$ & $45,1 \%$ & $54,9 \%$ & $100,0 \%$ \\
\hline & & & $\begin{array}{l}\% \text { de pacientes en base al } \\
\text { recuento de plaquetas }\end{array}$ & $100,0 \%$ & $100,0 \%$ & $100,0 \%$ \\
\hline \multirow[t]{9}{*}{ SÍ } & \multirow{6}{*}{$\begin{array}{l}\text { Aparición } \\
\text { de } \\
\text { metástasis }\end{array}$} & \multirow[t]{3}{*}{$\mathrm{NO}$} & Número de pacientes & 27 & 0 & 27 \\
\hline & & & $\begin{array}{l}\text { \% de pacientes dentro de los } \\
\text { pacientes sin metástasis }\end{array}$ & $100,0 \%$ & $0,0 \%$ & $100,0 \%$ \\
\hline & & & $\begin{array}{l}\% \text { de pacientes en base al } \\
\text { recuento de plaquetas }\end{array}$ & $96,4 \%$ & $0,0 \%$ & \\
\hline & & \multirow[t]{3}{*}{ SÍ } & Número de pacientes & 1 & 2 & 3 \\
\hline & & & $\begin{array}{l}\% \text { de pacientes dentro de los } \\
\text { pacientes con metástasis }\end{array}$ & $33,3 \%$ & $66,7 \%$ & $100,0 \%$ \\
\hline & & & $\begin{array}{l}\% \text { de pacientes en base al } \\
\text { recuento de plaquetas }\end{array}$ & $3,6 \%$ & $100,0 \%$ & \\
\hline & \multirow{3}{*}{\multicolumn{2}{|c|}{ Total }} & Número de pacientes & 28 & 2 & 30 \\
\hline & & & $\begin{array}{l}\% \text { de pacientes en base al } \\
\text { número de pacientes totales }\end{array}$ & $93,3 \%$ & $6,7 \%$ & $100,0 \%$ \\
\hline & & & $\begin{array}{l}\% \text { de pacientes en base al } \\
\text { recuento de plaquetas }\end{array}$ & $100,0 \%$ & $100,0 \%$ & $100,0 \%$ \\
\hline
\end{tabular}


Los resultados se agruparon en función de si los pacientes fueron tratados con terapia antiplaquetaria (azul) o no (amarillo) (Tabla 2). Posteriormente, se dividieron los pacientes en función de si presentaban o no metástasis y del recuento de plaquetas. La Tabla 2 muestra que, en el grupo de pacientes tratados con antiplaquetarios, el $90 \%$ de los pacientes no presentaron metástasis y presentaron recuento de plaquetas bajo (<450.000). Por otro lado, un $10 \%$ de los pacientes presentaron metástasis, teniendo el $66,7 \%$ recuento de plaquetas elevado $(\geq 450.000)$. Dentro del grupo de pacientes no tratados con antiplaquetarios el $59,8 \%$ no presentaron metástasis, de los cuales el $28,6 \%$ presentaron recuento de plaquetas alto ( $\geq 450.000$ ). Dentro del $40,2 \%$ que sí desarrolló metástasis, un $93.9 \%$ de los casos presentó recuento de plaquetas alto $(\geq 450.000)$.

Para demostrar que la correlación entre el recuento de plaquetas (lo que a su vez está condicionado por la existencia o no de tratamiento antiplaquetario) y la aparición de metástasis es estadísticamente significativa, realizamos el test chicuadrado a las 3 variables mencionadas, obteniendo un $\mathrm{p}$-valor $<0,05$.

Esto demuestra que la muestra analizada es suficientemente grande para obtener una significación estadística y que los estudios realizados corroboran el importante papel de las plaquetas en la progresión tumoral.

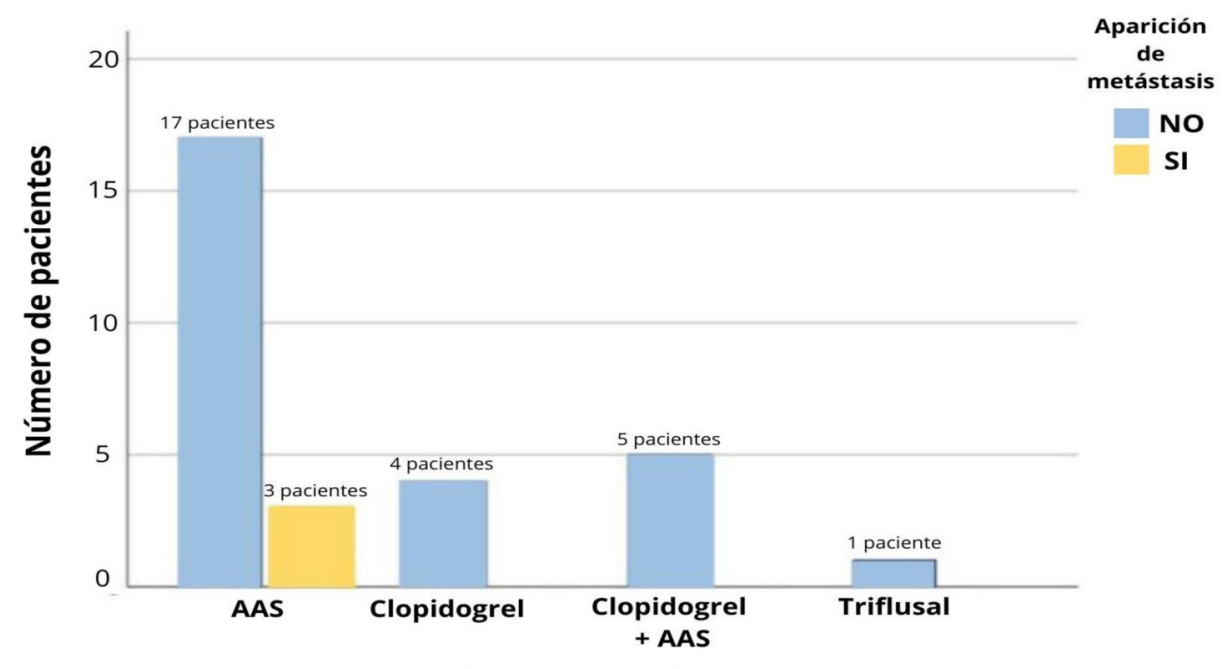

Medicamento antiplaquetario

Figura 1. Representación de la aparición de metástasis en función del tipo de medicamento. El histograma muestra el número de pacientes tratados con los distintos fármacos antiplaquetarios (azul) y, de ellos, cuántos desarrollaron metástasis (amarillo). En el caso de 
los tratados con Clopidogrel y Triflusal, ningún paciente desarrolló metástasis, pero esta muestra es muy pequeña comparada con la de pacientes tratados con AAS, por lo que no se pueden extraer conclusiones sobre la eficacia de los distintos tratamientos. AAS: aspirina.

También hemos analizado la eficacia de los diferentes fármacos antiplaquetarios en la inhibición de aparición de metástasis (Figura 1). Al aplicar la prueba de independencia chi-cuadrado a las variables medicamento antiplaquetario versus aparición de metástasis la correlación no fue significativa $(\mathrm{p}>0,05)$, posiblemente debido al bajo número de pacientes en algunas de las muestras (menor de 5 en varias de ellas). Sería necesaria una muestra mayor para obtener conclusiones significativas en cuanto a diferencias de eficacia de los diferentes fármacos.

\section{Discusión}

Los resultados de este trabajo muestran que el $90 \%$ de los pacientes de CCR tratados con antiplaquetarios no desarrollaron metástasis en comparación con el $59,8 \%$ de los pacientes sin terapia antiplaquetaria. Únicamente 3 pacientes (10\%) tratados con antiplaquetarios desarrollaron metástasis, de los cuales existían dudas sobre la adhesión terapéutica de uno de ellos.

De los pacientes que desarrollaron metástasis y no se encontraban realizando terapia antiplaquetaria, el 93,9\% (31 pacientes) presentaron alto recuento de plaquetas. Esto sugiere una correlación entre número de plaquetas y desarrollo de metástasis.

Por otra parte, entre los pacientes no sometidos a terapia antiplaquetaria y que no desarrollaron metástasis, el $28,6 \%$ presentó trombocitosis $(\geq 450.000)$ y, por tanto, sería de gran interés realizar el seguimiento de estos pacientes para la aparición de metástasis, en base al recuento elevado de plaquetas que presentan.

Son numerosos los estudios que defienden la reducción de la incidencia de cáncer tras el tratamiento con terapia antiplaquetaria, generalmente con AAS a una dosis $\geq 300 \mathrm{mg} /$ día. Por ejemplo, la reducción de aparición de metástasis tras el uso de terapia antiplaquetaria también ha sido demostrada por Loomans-Kropp et al., en cuyo estudio se describió la disminución de mortalidad en pacientes con neoplasia colorrectal que se encontraban bajo tratamiento antiplaquetario con AAS (Loomans-Kropp et al., 2019). Los resultados aquí presentados irían a favor de esta hipótesis.

Sin embargo, en otros estudios no hubo reducción en la incidencia de cáncer cuando los pacientes fueron tratados con terapia antiplaquetaria, por lo que existe controversia entre los distintos estudios realizados. De hecho, en dos estudios se menciona que existe un riesgo aumentado de desarrollo de cánceres en pacientes tratados con doble terapia antiplaquetaria con Clopidogrel/Prasugrel y AAS en 
comparación con pacientes tratados únicamente con AAS (Wiviott et al., 2007; Serebruany et al., 2017). Sin embargo, en dichos estudios no se compara la incidencia de cáncer con un grupo placebo. Tampoco se tiene en cuenta que la enzima que metaboliza los inhibidores del receptor plaquetario P2Y12 es la enzima CYP2C19, altamente polimórfica, y que el polimorfismo no metabolizador es más frecuente en población asiática, habiéndose realizado uno de los estudios con población coreana (Serebruany et al., 2017).

\section{BibliografíA}

Cedervall J, Hamidi A, Olsson AK. Platelets, NETs and cancer. Thromb. Res. 2018; 164: S148-S152.

Contursi A, Grande R, Dovizio M, Bruno A, Fullone R, Patrignani P. Platelets in cancer development and diagnosis. Biochem. Soc. Trans. 2018; 46(6):1517-1527.

Gasic GJ, Gasic TB, Stewart CC. Antimetastatic effects associated with platelet reduction. P. Nat. Acad. Sci. USA. 1968; 61(1):46-52.

Italiano JE, Richardson JL, Patel-Hett S, Battinelli E, Zaslavsky A, Short S et al. Angiogenesis is regulated by a novel mechanism: pro- and antiangiogenic proteins are organized into separate platelet $\alpha$ granules and differentially released. Blood. 2008; 111(3):1227-1233.

Ivanov II, Apta BHR, Bonna AM, Harper MT. Platelet P-selectin triggers rapid surface exposure of tissue factor in monocytes. Sci. Rep. 2019; 9(1):13397-13406.

Labelle M, Begum S, Hynes RO. Direct signaling between platelets and cancer cells induces an epithelial-mesenchymal-like transition and promotes metastasis. Cancer Cell. 2011; 20(5):576-590.

Levin J, Conley CL. Thrombocytosis associated with malignant disease. Arch. Intern. Med. 1964; 114(4):497-500.

Loomans-Kropp HA, Pinsky P, Cao Y, Chan AT, Umar A. Association of aspirin use with mortality risk among older adult participants in the prostate, lung, colorectal, and ovarian cancer screening trial. JAMA Netw. Open. 2019; 2(12): e1916729.

Menter DG, Tucker SC, Kopetz S, Sood AK, Crissman JD, Honn KV. Platelets and cancer: a casual or causal relationship: revisited. Cancer Metast. Rev. 2014; 33(1):231-269.

Placke T, Örgel M, Schaller M, Jung G, Rammensee HG, Kopp HG et al. Platelet-derived mhc class i confers a pseudonormal phenotype to cancer cells that subverts the antitumor reactivity of natural killer immune cells. Cancer Res. 2012; 72(2):440-448.

Serebruany VL, Kim MH, Cabrera-Fuentes HA, Lee K, Cho YR, Park K et al. Mortality and cancer after 12 versus 30 months dual antiplatelet therapy. Thromb. Haemost. 2017; 117(5):934-939.

Stoiber D, Assinger A. Platelet-leukocyte interplay in cancer development and progression. Cells. 2020; 9(4):855.

Suzuki-Inoue K. Platelets and cancer-associated thrombosis: focusing on the platelet activation receptor CLEC-2 and podoplanin. Blood. 2019; 134(22):1912-1918. 
Wiviott SD, Braunwald E, McCabe CH, Montalescot G, Ruzyllo W, Gottlieb S et al. Prasugrel versus clopidogrel in patients with acute coronary syndromes. New Engl. J. Med. 2007; 357(20):2001-2015.

Xu XR, Yousef GM, Ni H. Cancer and platelet crosstalk: opportunities and challenges for aspirin and other antiplatelet agents. Blood. 2018; 131(16):1777-1789.

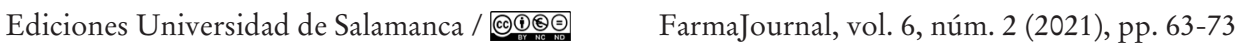


